\title{
Multiple Endocrine Neoplasia Type 1 Syndrome: A Case Report and Review of Literature
}

\author{
Hiya Boro ${ }^{1}$, Suraj Kubihal ${ }^{1}$, Saurabh Arora ${ }^{2}$, Vijay Kubihal ${ }^{3}$, Nikhil Tandon ${ }^{4}$ \\ 1. Endocrinology, Diabetes, and Metabolism, All India Institute of Medical Sciences, New Delhi, IND 2. Nuclear \\ Medicine, All India Institute of Medical Sciences, New Delhi, IND 3. Radiology, All India Institute of Medical Sciences,
} New Delhi, IND 4. Endocrinology and Metabolism, All India Institute of Medical Sciences, New Delhi, IND

Corresponding author: Hiya Boro, hiya21288@gmail.com

\begin{abstract}
Multiple endocrine neoplasia type 1 (MEN1) or Wermer's syndrome is a genetic disease characterized by involvement of multiple endocrine glands, primarily involving parathyroid, pancreas, and pituitary. Other additional features include foregut carcinoids; non-functioning adrenal tumors; and skin lesions such as lipomas, collagenomas, and angiofibromas. Here, we describe our experience in managing a patient who presented to us with spontaneous episodes of hypoglycemia and was diagnosed with insulinoma. Detailed clinical and biochemical evaluation unraveled the diagnosis of MEN1 in the patient and her family members who constituted a large kindred. This case highlights the importance for evaluation of MEN1 in a patient or his/her family members in the setting of clinical and biochemical suspicion. In addition, we have also discussed the utility of the latest diagnostic and therapeutic modalities for management of MEN1.
\end{abstract}

Categories: Endocrinology/Diabetes/Metabolism, Internal Medicine

Keywords: parathyroid adenoma, insulinoma, pancreatic net, pituitary adenoma

\section{Introduction}

Multiple endocrine neoplasia type 1 (MEN1) or Wermer's syndrome is a genetic disease caused by inactivating mutation of MEN1 gene. It is characterized by parathyroid, pituitary, and pancreatic neoplasms. In addition, other features include foregut carcinoids; non-functioning adrenal tumors; skin lesions such as lipomas, angiofibromas, and collagenomas. Most of the mutations are germline, but sporadic and de novo mutations can also occur. The disease affects all age groups from five to 80 years. Most of the manifestations are evident by the fifth decade of life. The clinical manifestations of MEN1 are related to the tumor site and the hormonal products released. In the absence of treatment, mortality rate is $50 \%$ by 50 years of age [1].

Review began $11 / 26 / 2020$ Review ended 12/11/2020 Published 12/14/2020

\section{๑) Copyright 2020}

Boro et al. This is an open access article distributed under the terms of the Creative Commons Attribution License CC-BY 4.0., which permits unrestricted use, distribution, and reproduction in any medium, provided the original author and source are credited.
Here, we describe our experience with a case of MEN1 syndrome who had presented with one predominant organ-related symptom. Subsequently, on evaluation she was found to harbor multiple tumors. She was the index case of a large kindred, and her diagnosis brought to attention the presence of MEN1 in other family members.

\section{Case Presentation}

A 46-year-old female presented to us with multiple episodes of increased hunger, diaphoresis, palpitations, tremors, anxiety, and dizziness for the last eight months. These episodes usually occurred in the fasting state. During one such episode, her blood glucose recorded by glucometer was $35 \mathrm{mg} / \mathrm{dl}$. Her symptoms were reversed by intake of carbohydrate diet. Though Whipple's triad was not established as plasma glucose was not measured, the symptomatology did merit for further evaluation [2]. She had no history of diabetes, chronic liver disease, chronic kidney disease, or history of intake of any drug known to cause hypoglycemia.

On further probing, she revealed history of recurrent renal stones for the past 16 years. In addition, she had history of galactorrhea and oligomenorrhea for the past two years. Apart from this, there was no other significant history. She was the index case in her family. However, her family history did reveal that she was part of a large kindred. She had five siblings and 22 step siblings from the five marriages of her father. Patient's father who had similar episodes of neuroglycopenic symptoms [suspected (?) insulinoma] and history of renal stones (Figure 1) had expired before evaluation. There was history suggestive of at least one MEN1-associated endocrine tumor among eight of her siblings. The pedigree chart is shown in Figure 1. 


\section{Cureus}

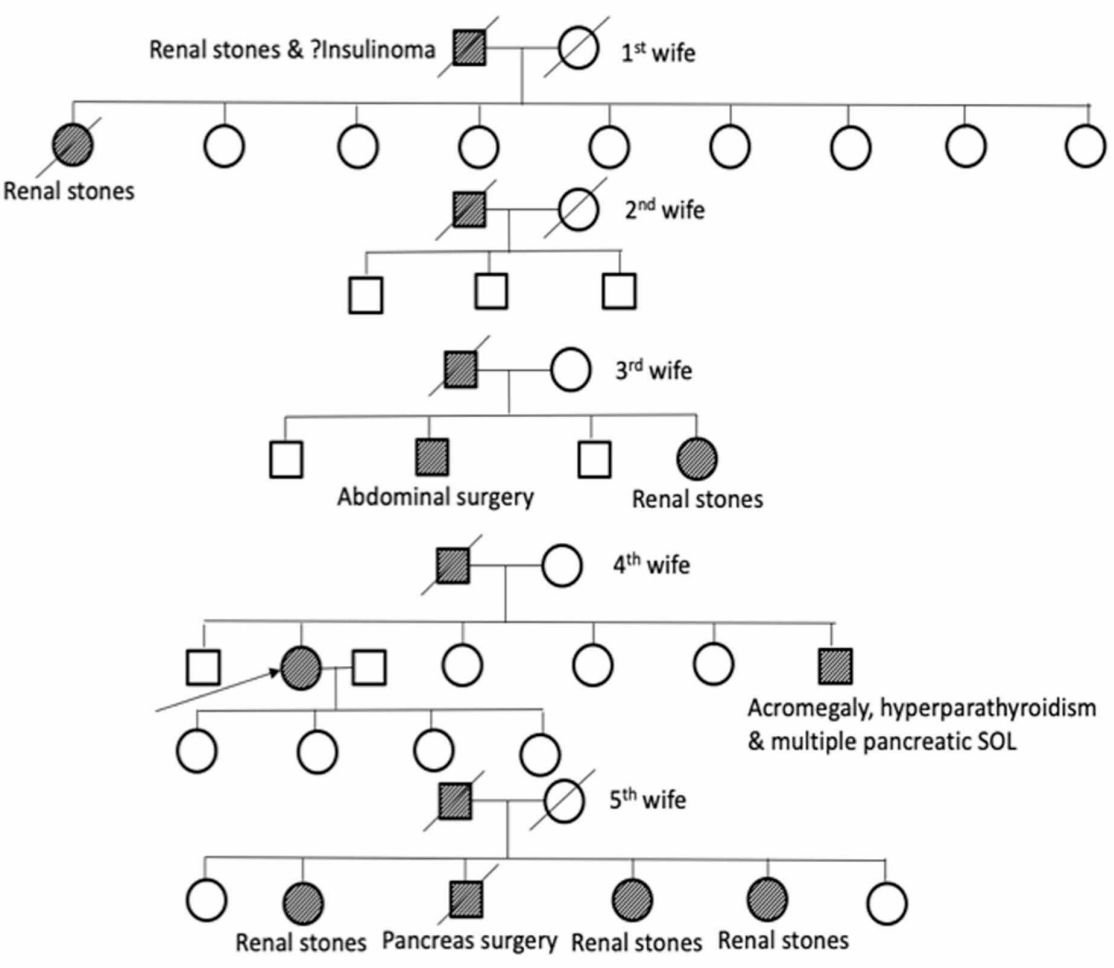

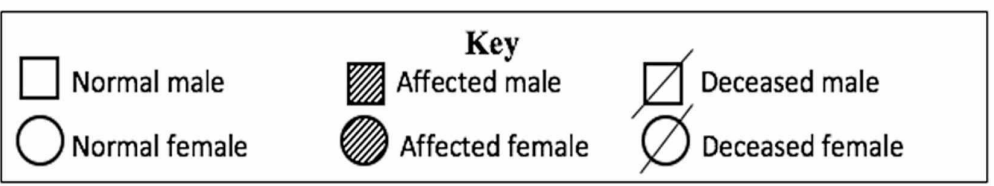

\section{FIGURE 1: Pedigree chart of our patient}

SOL, Space-occupying lesion; (?) insulinoma-suspected insulinoma

After admission in our ward, the patient had an episode of spontaneous hypoglycemia, and critical sample was analyzed that revealed non-ketotic hyperinsulinemic hypoglycemia (blood glucose of $40 \mathrm{mg} / \mathrm{dl}$ with corresponding serum insulin $19.4 \mu \mathrm{U} / \mathrm{ml}$ and C peptide of $4.9 \mathrm{ng} / \mathrm{ml}$, blood ketones of $0.1 \mathrm{mmol} / \mathrm{L}$ ) [3]. Serum insulin and C peptide were measured by electrochemiluminescence (ECLIA) assay (Cobas e411, Roche diagnostics, Germany), while blood ketones were measured on a finger prick test using a point of care device (normal range 0.4-0.5 mmol/L). Patient also had parathyroid hormone (PTH)-dependent hypercalcemia with hypercalciuria [serum total calcium $12.3 \mathrm{mg} / \mathrm{dl}$ (normal range: 8.5-10.5 mg/dl), serum intact PTH of 210 $\mathrm{pg} / \mathrm{ml}$ (normal range 15-65 pg/ml), 24-hour urinary calcium of $450 \mathrm{mg} /$ day (normal range < $250 \mathrm{mg} /$ day)]. Serum calcium was measured on Roche Hitachi 917 (automated analyzer), while serum intact PTH was measured on ECLIA (minimum detection limit $1.5 \mathrm{pg} / \mathrm{ml}$ ). Her serum prolactin level, measured by ECLIA, was also elevated, $92.6 \mathrm{ng} / \mathrm{ml}$ (normal range, 10-29 ng/ml). Other pituitary hormones were within normal limits.

A suspicion of MEN1 syndrome was made, which was later confirmed by positive screening test for MEN1 gene mutation (Deletion: c.824-832delGGTACCCCA in exon 5 of MEN1 gene on whole gene sequencing).

For evaluation of PTH-dependent hypercalcemia, she underwent technetium-99m SESTAMIBI scan that revealed involvement of three parathyroid glands (left superior, left inferior, and right paratracheal) (Figures $2 A, 2 B$ ). However, the four-dimensional computed tomography (4D CT) (Figure 2C) and four-dimensional magnetic resonance imaging (4D MRI) (Figure 2D) showed involvement of all the four glands. 


\section{Cureus}
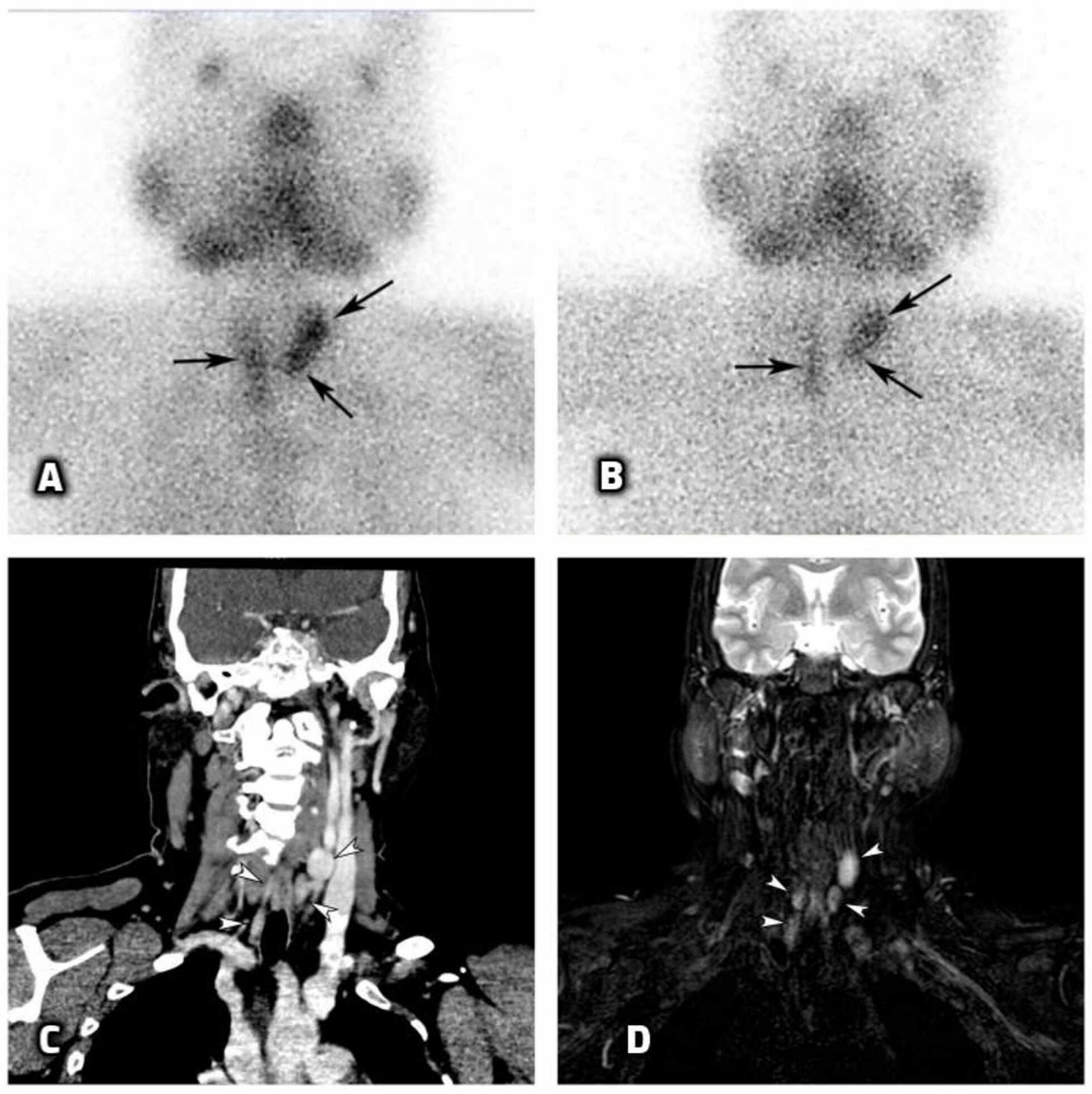

FIGURE 2: Technetium-99m SESTAMIBI (A, B), 4D CT (C), and 4D MRI (D) scans of the patient showing parathyroid adenomas

Early (A) and delayed (B) phase images of SESTAMIBI scan showing elongated areas of early increased tracer uptake in right inferior, left superior, and left inferior parathyroid gland, persisting in delayed phase.

Coronal venous phase image of 4D CT (C) and coronal T2-weighted 4D MRI image (D) showing involvement of all four parathyroid glands.

For evaluation of hyperinsulinemic hypoglycemia, she underwent multiphase contrast-enhanced computed tomography (CT) of abdomen that revealed two mass lesions in the pancreatic head and tail, respectively (Figures 3A, 3B).
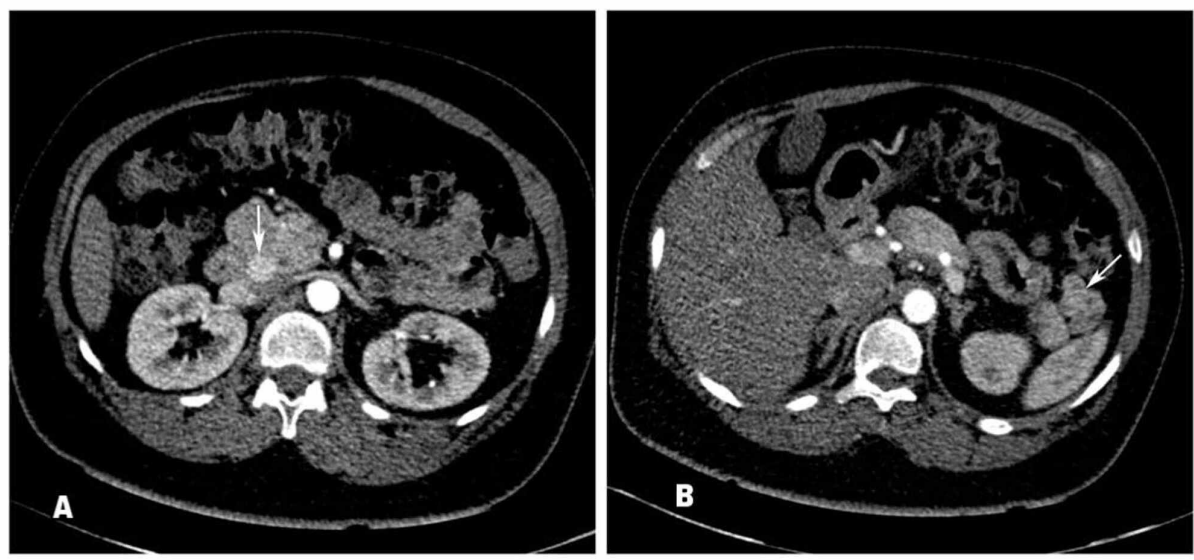

FIGURE 3: CT images showing pancreatic NET

Axial pancreatic phase images of multiphase contrast-enhanced CT images show hyper-enhancing nodule in 


\section{Cureus}

the pancreatic head $(A)$ and another heterogeneous exophytic lesion with eccentric nodular

hyperenhancement in the pancreatic tail region (B) s/o pancreatic neuroendocrine tumor (NET).

Patient also underwent Ga-68-DOTANOC positron emission tomography (PET)/computed tomography (CT) that revealed somatostatin receptor (SSTR) expressing lesions in the head of the pancreas $(1.6 \mathrm{~cm} \times 1.5 \mathrm{~cm})$ and tail of the pancreas $(3.2 \mathrm{~cm} \times 2.2 \mathrm{~cm}$ ) (Figures $4 A-4 C)$. She also underwent endoscopic ultrasonography (EUS) that revealed multiple hypoechoic round lesions in the pancreas (max: $3.5 \mathrm{~cm} \times 2.1 \mathrm{~cm} \times 0.8 \mathrm{~cm}$ ) with vascularity in the lesions, two lesions of diameter $2.1 \mathrm{~cm}$ and $1.1 \mathrm{~cm}$ in the body of pancreas, and four small lesions in the tail of pancreas ( $8 \mathrm{~mm}$ each).

A

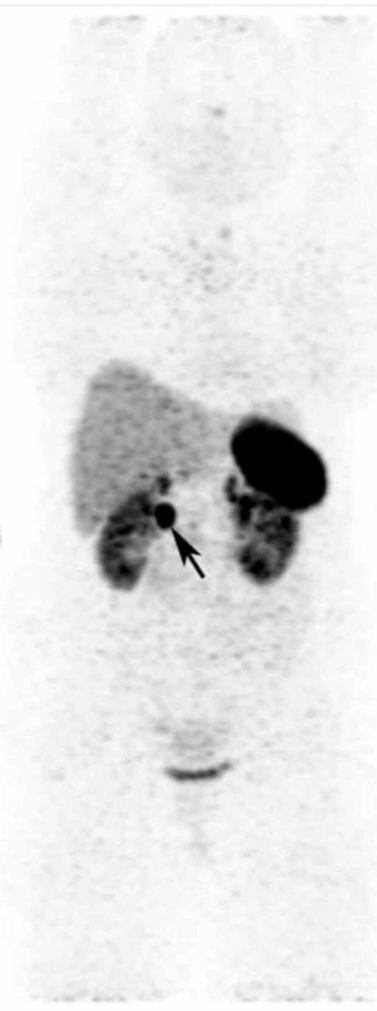

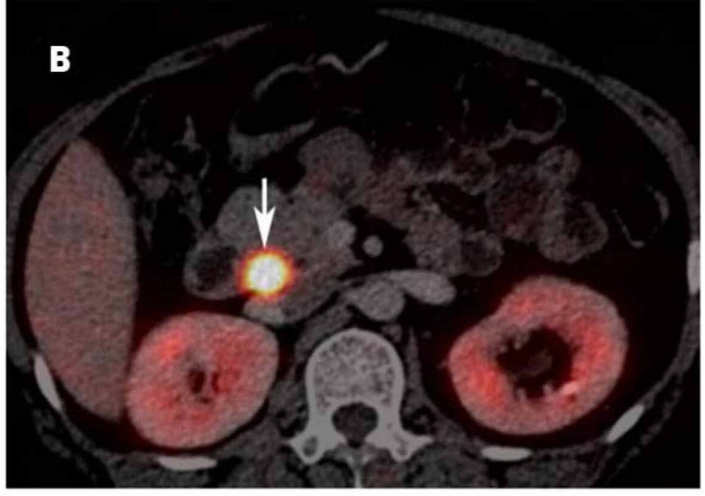

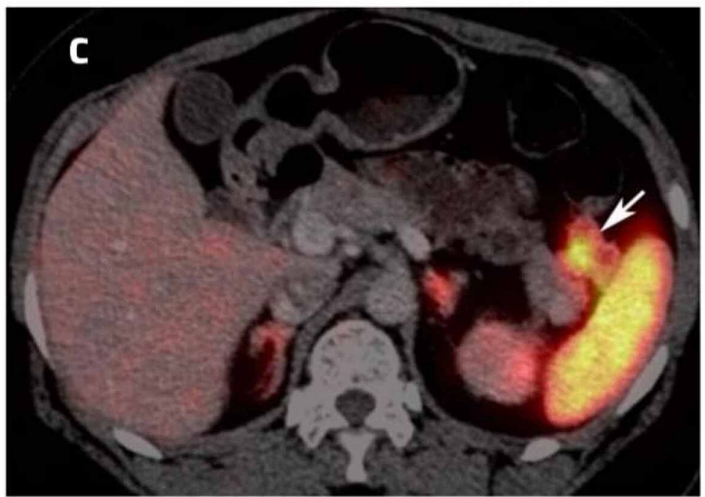

\section{FIGURE 4: Ga-68-DOTANOC scan images of the patient}

Maximum intensity projection (MIP) image (A) and axial fused positron emission tomography/computed tomography (PET/CT) images (B and $\mathrm{C}$ ) show two somatostatin receptor expressing lesions in the pancreatic head $(1.6 \mathrm{~cm} \times 1.5 \mathrm{~cm})(\mathrm{B})$ and tail region $(3.2 \mathrm{~cm} \times 2.2 \mathrm{~cm})(\mathrm{C})$, respectively.

For evaluation of pituitary disease, she underwent an MRI sella that revealed a 9-mm pituitary adenoma (Figures $5 A, 5 B$ ).
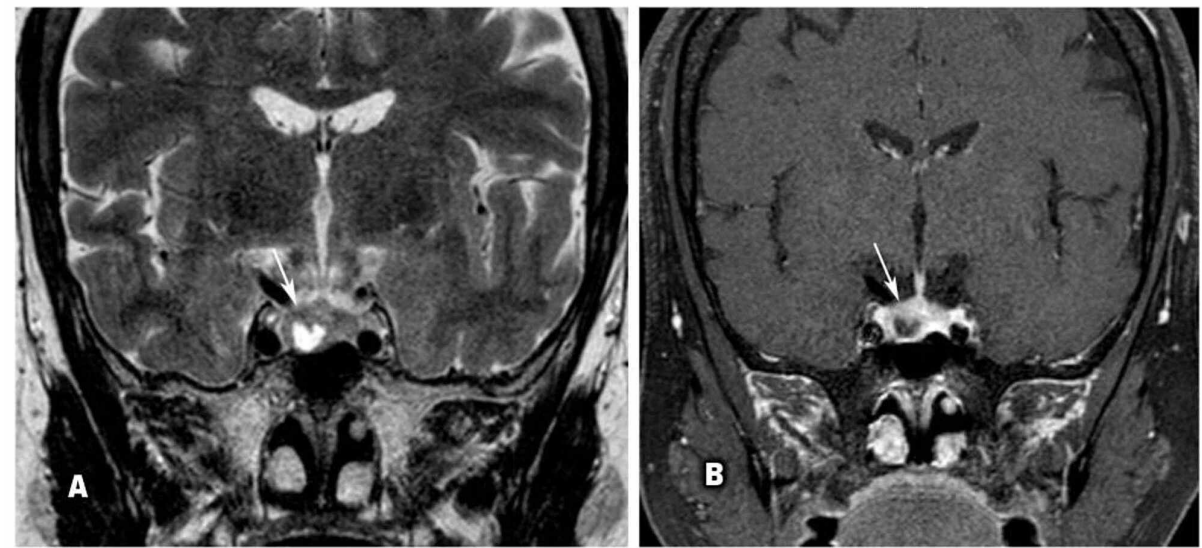
She underwent removal of all parathyroid glands, cervical thymectomy, and auto-transplantation of normal parathyroid tissue into left forearm. Postoperatively, she was managed with calcium supplements and calcitriol. She also underwent total pancreatico-duodenectomy along with splenectomy. Post-surgery, glycemic control was achieved with insulin. Exocrine functions were managed with pancreatic enzyme supplements. For prolactinoma, she was started on tablet cabergoline $0.5 \mathrm{mg}$ twice a week.

Histopathology of parathyroid specimens revealed parathyroid hyperplasia, while that of pancreatic specimen revealed well-differentiated neuroendocrine neoplasm, grade 1 (G1), with multiple multicentric microadenomas (pathological stage: pT2N0). Patient was counseled regarding the importance of regular follow-up. Investigations and their intervals were planned as defined for MEN1 patients [4].

Her brother, who was a 42-year-old male, also had symptoms suggestive of spontaneous hypoglycemia corrected by intake of carbohydrate diet and acromegaloid habitus. He too was admitted for evaluation and was found to have acromegaly with pituitary macroadenoma, primary hyperparathyroidism with adenomas in all the four parathyroid glands, and hyperinsulinemic hypoglycemia with multiple insulinomas. He too underwent total parathyroidectomy along with auto-transplantation of normal parathyroid tissue in nondominant forearm, with cervical thymectomy. For pituitary macroadenoma, he underwent trans-nasal transsphenoidal surgery (TNTS) with uneventful recovery. However, he refused to undergo pancreatic surgery and instead resorted to medical therapy with frequent, low carbohydrate meals.

\section{Discussion}

MEN1 is usually inherited as an autosomal dominant condition with a high degree of penetrance but can also be sporadic [5]. MEN1 can be diagnosed clinically when a patient presents with two or more MEN1associated tumors (parathyroid adenomas, entero-pancreatic tumors, and pituitary adenomas). Diagnosis can be familial when a patient has a MEN1-associated tumor and a first degree relative with MEN1 [6]. Diagnosis can also be genetic when a person has MEN1 mutation without evident clinical or biochemical characteristics [6]. Commonly penetrated tumors in MEN1 are parathyroid adenomas (90\%), enteropancreatic tumors (30\%-70\%), and pituitary adenomas (30\%-40\%).

In our patient, the presenting complaint was that of hyperinsulinemic hypoglycemia, which was evaluated and diagnosed to be a case of multiple insulinomas. However, on detailed history she also had complaints directing toward involvement of other endocrine glands. Subsequently, she was evaluated and found to have primary hyperparathyroidism (PHPT) and prolactinoma. This case underlines the importance to look for symptomatology or biochemical clues suggestive of MEN1 syndrome when the patient presents with one of the MEN1-associated endocrine tumors. She, however, did not have other traditional hallmarks of MEN1 such as lipoma, collagenoma, angiofibroma, foregut carcinoid, or adrenal lesion.

Pre-operative imaging of pancreatic NET (neuro-endocrine tumor) not only aids in the diagnosis but also improves the success rate of surgery. Our patient underwent a structural imaging (triple-phase CT abdomen) as well as a functional imaging (Ga-68-DOTANOC study) for the localization of insulinoma. However, in MEN1, concurrent pancreatic NETs pose a challenge for the correct localization of the insulinoma.

Endoscopic ultrasound remains a valuable pre-operative diagnostic tool for detection of pancreatic tumors, even up to 1-2 mm, which are otherwise undetectable by CT/MRI [7]. It is also challenging to differentiate between functioning and non-functioning pancreatic NET. Selective intra-arterial calcium stimulation test with hepatic venous gradient for insulin and C peptide can facilitate precise localization of insulinomas and can help differentiate between functioning and non-functioning pancreatic tumors [8]. In our patient, due to concomitant hypercalcemia, she was not subjected to intra-arterial calcium stimulation test.

While evaluating for PTH-dependent hypercalcemia, technetium-99m SESTAMIBI scan revealed adenomas in three parathyroid glands. On the contrary, 4D CT and 4D MRI revealed adenomas in all four glands. This does highlight the pitfall of technetium-99m SESTAMIBI scan as it provides false negative results in case of parathyroid hyperplasia or multiple parathyroid adenomas [9]. In MEN1 syndrome, pre-operative localization of parathyroid lesion is of limited benefit, and surgical exploration would be required irrespective of pre-operative imaging [4]. There has been controversy regarding the most preferred surgical approach of PHPT in MEN1. A few surgeons prefer subtotal parathyroidectomy, while others advocate total parathyroidectomy with auto-transplantation of normal parathyroid tissue into non-dominant forearm. Cervical thymectomy is also recommended along with parathyroidectomy in MEN1. Our patient had involvement of all four glands, hence was subjected to the latter surgical procedure. Measurement of intraoperative PTH by Turbo PTH assay and fulfillment of Miami criterion (more than 50\% decline in serum 


\section{Cureus}

PTH from the pre-incision value at 10 minutes post excision) $[10,11]$ can help provide an idea about the immediate success of surgery.

MRI of the sella turcica revealed pituitary adenoma in our patient. Prolactinomas are the most common pituitary tumors in MEN1, and the same was observed in our patient, corroborating with clinical and biochemical presentation.

Clinical diagnosis of MEN1 was confirmed by genetic analysis in our patient. MEN1 gene on chromosome 11q13 encodes a 610-amino acid protein termed menin. Menin mediates stimulatory or inhibitory effects on numerous transcription factors by epigenetic mechanisms. Menin may act as a tumor suppressor or an oncogene depending upon its interaction with different proteins. Many proteins involved in DNA damagedependent cell cycle arrest or subsequent DNA damage repair are dependent on the co-presence of functional menin [12].

Diagnosis of MEN1 and further confirmation by genetic analysis have many implications. MEN1-associated tumors differ from non-syndromic tumors in various aspects that are enlisted in Tables 1-3. These differences along with the need for comprehensive management and surveillance in MEN1 signify the importance of management of these patients by a multidisciplinary team.

\begin{tabular}{|l|l|l|}
\hline & MEN1 & Non-MEN1 \\
\hline Age of onset (years) & 25 & 55 \\
\hline Female: male ratio & $1: 1$ & $3: 1$ \\
\hline Biochemistry & Borderline rather than elevated PTH levels Mildly elevated serum calcium & $\begin{array}{l}\text { Elevated PTH and } \\
\text { calcium levels }\end{array}$ \\
Reduction in BMD & Greater & Lesser \\
Pathology & Multiglandular hyperplasia & Solitary adenoma \\
Surgery & Subtotal parathyroidectomy (at least 3.5 glands) or total parathyroidectomy with & $\begin{array}{l}\text { Surgical removal of } \\
\text { the abnormal gland }\end{array}$ \\
Intraoperative PTH & More useful & Useful \\
$\begin{array}{l}\text { Post-operative rate of } \\
\text { hypoparathyroidism }\end{array}$ & Higher & Lower \\
Recurrence rates & High & Low \\
Parathyroid cancer & Almost never progresses to parathyroid cancer & Might \\
\hline
\end{tabular}

\section{TABLE 1: Differences between PHPT in MEN1 and non-MEN1 (sporadic)}

PHPT, primary hyperparathyroidism; MEN1, multiple endocrine neoplasia type 1; PTH, parathyroid hormone; BMD, bone mineral density. 


\section{Cureus}

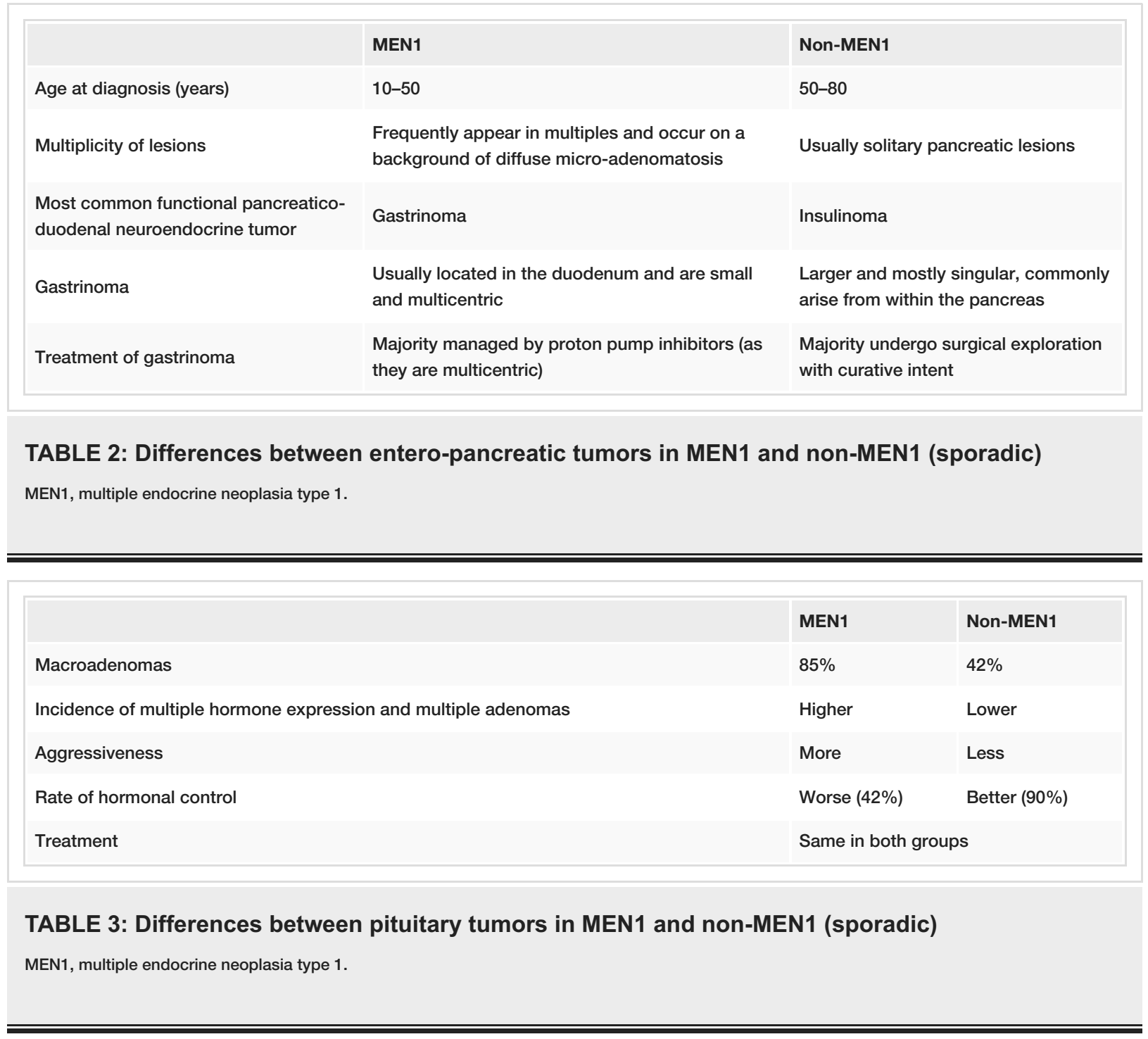

\section{Conclusions}

We have described a case of multiple endocrine neoplasia type 1 (MEN1) syndrome who presented to us with episodes of spontaneous hypoglycemia. The diagnosis of MEN1 syndrome in our patient led to the discovery of a large kindred being affected with the same disease. This underlies the importance for searching for MEN1-related tumors and genetic screening in family members on the basis of clinical and biochemical suspicion.

\section{Additional Information}

\section{Disclosures}

Human subjects: Consent was obtained by all participants in this study. Conflicts of interest: In compliance with the ICMJE uniform disclosure form, all authors declare the following: Payment/services info: All authors have declared that no financial support was received from any organization for the submitted work. Financial relationships: All authors have declared that they have no financial relationships at present or within the previous three years with any organizations that might have an interest in the submitted work. Other relationships: All authors have declared that there are no other relationships or activities that could appear to have influenced the submitted work.

\section{References}

1. Wilkinson S, Teh BT, Davey KR, McArdle JP, Young M, Shepherd JJ: Cause of death in multiple endocrine neoplasia type 1. Arch Surg. 1993, 128:683-90. 10.1001/archsurg.1993.01420180085016

2. Whipple AO: The surgical therapy of hyperinsulinism. J Int Chir. 3:237-276.

3. De León DD, Stanley CA: Determination of insulin for the diagnosis of hyperinsulinemic hypoglycemia . Best Pract Res Clin Endocrinol Metab. 2013, 27:763-9. 10.1016/j.beem.2013.06.005

4. Thakker RV, Newey PJ, Walls GV, et al.: Clinical practice guidelines for multiple endocrine neoplasia type 1 (MEN1). J Clin Endocrinol Metab. 2012, 97:2990-3011. 10.1210/jc.2012-1230 


\section{Cureus}

5. Turner JJ, Christie PT, Pearce SH, et al.: Diagnostic challenges due to phenocopies: lessons from multiple endocrine neoplasia type 1 (MEN1). Hum Mutat. 2010, 31:1089-1101. 10.1002/humu.21170

6. Falchetti A: Genetics of multiple endocrine neoplasia type 1 syndrome: what's new and what's old . F1000Res. 2017, 6:73. 10.12688/f1000research.7230.1

7. Kann PH, Balakina E, Ivan D, et al.: Natural course of small, asymptomatic neuroendocrine pancreatic tumours in multiple endocrine neoplasia type 1: an endoscopic ultrasound imaging study. Endocr Relat Cancer. 2006, 13:1195-202. 10.1677/erc.1.01220

8. Won JGS, Tseng H-S, Yang A-H, et al.: Intra-arterial calcium stimulation test for detection of insulinomas: detection rate, responses of pancreatic peptides, and its relationship to differentiation of tumor cells. Metabolism. 2003, 52:1320-9. 10.1016/s0026-0495(03)00200-2

9. Chen EM, Mishkin FS: Parathyroid hyperplasia may be missed by double-phase Tc-99m sestamibi scintigraphy alone. Clin Nucl Med. 1997, 22:222-6. 10.1097/00003072-199704000-00002

10. Barczynski M: Minimally invasive parathyroidectomy without intraoperative parathyroid hormone monitoring: when and why?. J Postgrad Med. 2009, 55:239-240. 10.4103/0022-3859.58923

11. Sokoll LJ, Wians FH, Remaley AT: Rapid intraoperative immunoassay of parathyroid hormone and other hormones: a new paradigm for point-of-care testing. Clini Chem. 2004, 50:1126-35. 10.1373/clinchem.2003.030817

12. Agarwal SK: The future: genetics advances in MEN1 therapeutic approaches and management strategies . Endocr Relat Cancer. 2017, 24:119-34. 10.1530/ERC-17-0199 